\title{
Las TIC: Una herramienta tecnológica para el desarrollo de las competencias lingüísticas en estudiantes universitarios de una segunda lengua
}

\author{
ICT as a Technological Tool for the Development of Language Skills in \\ Second Language University Students
}

\author{
Ginneth Pizarro Chacón ${ }^{7}$ \\ Universidad Nacional \\ Heredia, Costa Rica \\ ginneth.pizarro.chacon@una.cr \\ Damaris Cordero Badilla² \\ Escuela de Literatura y Ciencias del Lenguaje \\ Universidad Nacional \\ Heredia, Costa Rica \\ Jodaro.damaris@gmail.com
}

Recibido 28 de junio de 2012 • Corregido 10 de junio de 2013 • Aceptado 19 de junio de 2013

Resumen. Este trabajo tuvo como objetivo investigar y recolectar información relacionada con el uso de las TIC por parte de profesores de lengua de la Escuela de Literatura y Ciencias del Lenguaje de la UNA y cómo estas herramientas tecnológicas son incorporadas en el currículo de la clase. Además, aporta algunas ideas sobre estrategias metodológicas que se pueden utilizar en el salón de clase con el uso de TIC. Se realizó una investigación descriptiva, de campo, con un enfoque cualitativo. Se recurrió al paradigma cualitativo interpretativo, también llamado fenomenológico, naturalista, humanista o etnográfico, porque se centra en comprender e interpretar la realidad, los significados y las intenciones de las personas, con el fin de construir conocimiento. El estudio se lleva a cabo en un contexto real; se utilizó un cuestionario que fue aplicado a 21 profesores de lengua de la ELCL. Los resultados obtenidos evidenciaron el poco uso de estas herramientas tecnológicas por parte de los docentes en sus salones de clase. Además, se mostró la necesidad de una mayor capacitación para conocer, aprender e incorporar estas herramientas en el currículo, lo cual permite que se desarrolle un proceso de enseñanza y aprendizaje activo y efectivo. Finalmente, las investigadoras aportaron una lista de recomendaciones sobre técnicas de enseñanza que ayuden al profesorado a mejorar sus habilidades para incorporar estas herramientas en sus actividades docentes.

1 Máster en Lingüística de la Universidad de Costa Rica. Labora como docente impartiendo los cursos de Lingüística en la Escuela de Literatura y Ciencias del Lenguaje de la UNA. Ha realizado trabajos de investigación en gramática comunicativa, sociolingüística, lenguas indígenas, criollo limonense, uso de TIC en la enseñanza y técnicas metodológicas en la enseñanza de una segunda lengua, entre otros. Ha realizado publicaciones en varias revistas.

2 Máster en Educación con énfasis en la Enseñanza del Inglés de la Universidad Latina de Costa Rica. Y Máster en Administración Universitaria (M.A.U) de la Universidad de Costa Rica. Labora como docente en la Escuela de Literatura y Ciencias del Lenguaje de la UNA. Ha realizado trabajos de investigación en gramática comunicativa, estrategias metodológicas innovadoras y los usos de TIC en la docencia universitaria, entre otros. Ha publicado artículos sobre estos temas en varias revistas nacionales e internacionales. 
URL: http://www.una.ac.cr/educare

CORREO: educare@una.cr

Palabras claves. Tecnologías de información y comunicación (TIC), competencias lingüísticas, clase de lengua, estrategias de enseñanza, recursos tecnológicos.

Abstract. This work aimed to make a research and collect information related to the use of ICTs by teachers of language at the School of Literature and Language Sciences (ELCL) of the Universidad Nacional (Costa Rica) and how they incorporate technological tools into the curriculum of their classes. It also provides some ideas on methodological strategies that can be used in the classroom with the use of ICT. To achieve the main objectives of this work, a questionnaire was used to conduct the research from a descriptive point of view, field work, through a qualitative approach. The interpretive/ qualitative paradigm, also called phenomenological, naturalist, humanist or ethnographic, was used since it focuses on understanding and interpreting people's reality, meanings and intention in order to build knowledge. The study takes place in a real context and a questionnaire was applied to 21 ELCL language teachers. The findings revealed that language teachers incorporate technological tools in a few activities in their classrooms; they do not take advantage of their applicability in all class activities. Besides, they really need more training to know, learn, and incorporate these tools in their curriculum, so they will develop an effective teaching and an active learning. This directed researchers to provide a list of recommendations about teaching techniques that will help language teachers improve their skills and incorporate these tools into their teaching strategies.

Keywords. Information Communication Technology (ICT), language skills, language class, teaching strategies, technological tools.

Son muchos los beneficios que las tecnologías de información y comunicación (TIC) ofrecen en los contextos de educación superior; estas herramientas engrandecen la calidad del proceso educativo, y permiten la superación de barreras de espacio y tiempo, una mayor comunicación e interacción entre el profesorado y el alumnado, una participación activa en el proceso de construcción colectiva del conocimiento y el desarrollo de habilidades.

Con ellas, tanto el estudiantado como el personal docente de los programas de enseñanza y aprendizaje de una segunda lengua tienen la posibilidad de una mayor interacción con estudiantes y profesorado de otros programas, instituciones y lugares tanto nacionales como internacionales. Esto brinda la oportunidad de la construcción del conocimiento con el uso de contenidos y material didáctico actualizado, así como del acceso a una cantidad incalculable de fuentes de información proporcionadas por Internet.

\section{Estado de la cuestión o antecedentes}

Para enfrentar los retos que las instituciones de educación superior tienen en la actualidad, se deben promover experiencias innovadoras en los procesos de enseñanza y aprendizaje apoyados en las TIC, así como grandes cambios en las estrategias didácticas del profesorado y en el tipo de materiales de aprendizaje que se manejan en la labor docente (Salinas, 2004).

En un estudio hecho por Fernández, Server y Carballo (2006), se evidencia que, con la incorporación de TIC en la enseñanza, ".. . el alumno se transforma en participante activo y constructor de su propio aprendizaje y el profesor asume el rol de guía y facilitador ... lo cual varía su forma de 
interactuar con sus alumnos, la forma de planificar y de diseñar el ambiente de aprendizaje (p. 1). Así mismo, estas herramientas tecnológicas permiten que el personal docente encuentre técnicas innovadoras para estimular la motivación del estudiantado y mejorar su aprendizaje con el uso de multimedia, hipertextos y otros sistemas interactivos, además de elegir aquellos recursos didácticos que mejor convengan a las distintas áreas del conocimiento de los estudiantes, tales como: aulas virtuales, videoconferencias, y búsqueda de información actualizada, entre otros.

Ya desde los años 60, estas innovadoras herramientas informáticas han afectado la enseñanza de lenguas extranjeras, han contribuido a desarrollar una metodología comunicativa, y han ayudado a implementar las primeras actividades sencillas de gramática, sintaxis y traducción con el uso del computador. Pero es a partir de los años 70 cuando se demuestra un mayor interés en investigar las ventajas de estas herramientas como medio de enseñanza y aprendizaje (Manga, 2008).

No se debe olvidar que en la actualidad la pedagogía lingüística en la enseñanza de una segunda lengua apoya el uso de una metodología comunicativa en donde se adquiera la lengua de manera práctica y natural. Estas características han sido imitadas por las TIC en condiciones de uso natural mediante el empleo de herramientas tales como el correo electrónico, el envió de archivos adjunto de texto, imágenes, videos o sonidos, las salas de chat, los foros, las listas de distribución (mailing-lists), y las videoconferencias, entre otras, para facilitar el aprendizaje (Contreras, 2008).

Además, tradicionalmente en la enseñanza de una segunda lengua se han aplicado sistemas multimedia; sin embargo, desde que existen las TIC, los docentes disponen de herramientas más innovadoras para motivar al estudiantado. De esta forma se complementa la naturaleza interactiva del proceso y aprendizaje de una segunda lengua, por medio de la interacción del profesor y del uso de nuevas experiencias de enseñanza y aprendizaje en el salón de clase.

\section{Referentes conceptuales}

UNESCO (2004) en Las tecnologías de información y comunicación en la formación docente: Guía de planificación, evidencia cómo en años recientes han surgido nuevas formas de concebir el proceso de aprendizaje como un aprendizaje centrado en el alumno. Estas se han apoyado en investigaciones hechas sobre teorías relacionadas con el contexto del aprendizaje. Teorías tales como la teoría sociocultural, la constructivista, el aprendizaje regulado, la cognición situada, el aprendizaje cognitivo, el aprendizaje basado en resolución de problemas, entre otras, se basan en el criterio de que los estudiantes y las estudiantes son agentes activos que buscan y construyen el conocimiento con un propósito y dentro de un contexto significativo.

Desdeesta premisa, lasTIC constituyen herramientas que ayudan a los estudiantes a acceder a variados recursos de conocimientos; a colaborar y compartir con sus compañeros; a resolver problemas utilizando diferentes herramientas epistemológicas; a desarrollar comunidades de 
URL: http://www.una.ac.cr/educare

CORREO: educare@una.cr

aprendizaje integradas por otros estudiantes, docentes y expertos, y a participar en grupos de discusión o debates (UNESCO, 2004).

En la enseñanza de una segunda lengua en particular, se emplean las TIC al usar videos para facilitar la colaboración online como forma de apoyar la construcción de conocimiento colaborativo, desarrollar materiales, implementar aprendizaje a distancia, desarrollar habilidades metacognitivas y convertir al estudiantado en personas más reflexivas y autorreguladas (Hsiao, 1999, citado por UNESCO, 2004).

Tal es el caso de las TIC en la enseñanza del inglés, que al ser estos medios interactivos y colaborativos permiten al estudiantado explorar el lenguaje de manera más creativa, tener la oportunidad de acceder y organizar la información de forma variada, y desarrollar la comprensión de la segunda lengua de forma crítica.

Las TIC en la enseñanza de un idioma ofrecen la oportunidad de usar la lengua extranjera de manera significativa en contextos auténticos, suministran un acceso fácil y rápido para el uso de materiales reales y actualizados en la lengua estudiada, y presentan oportunidades motivadoras de aprendizaje tales como las salas de chat o los ambientes virtuales (Vélez, Vidal y Yamá, 2010). Por esto, los docentes en la enseñanza de una segunda lengua también se ven beneficiados con el uso de estas herramientas tecnológicas, pues cuentan con un gran número de recursos educativos disponibles, lo que les ayudaría a usar enfoques diferentes de enseñanza y aprendizaje con cada estudiante y sus distintos estilos de aprendizaje.

\section{Metodología, propósito del estudio y preguntas de la investigación}

Para el desarrollo del presente trabajo se realizó una investigación exhaustiva de información relacionada con el tema en cuestión y, simultáneamente, se elaboró un cuestionario para ser entregado a diecinueve docentes del Bachillerato en la Enseñanza del Inglés y del Bachillerato en la Enseñanza del Francés, en la Escuela de Literatura y Ciencias del Lenguaje de la Universidad Nacional. En él se incluyó una pregunta cerrada y diez preguntas abiertas relacionadas con el uso pedagógico de las TIC en la enseñanza de una lengua, en aspectos tales como: el empleo de TIC y la forma en que estas herramientas tecnológicas facilitan los procesos de enseñanza y aprendizaje de una segunda lengua, la opinión sobre si el profesor de lengua debe adaptar sus métodos de enseñanza al manejo de TIC en los cursos, la forma en que utilizan algunos recursos tecnológicos en la clase, la inclusión del uso de TIC en la planeación de los cursos, el nombre de los cursos en los cuales aplica TIC para la enseñanza, actividades que realiza en la clase de lengua con el uso de TIC, opiniones sobre la forma en que el empleo de TIC en los cursos modifica las estrategias de enseñanza, ventajas de utilizar TIC en una clase de lengua y sobre el cambio en las competencias lingüísticas al servirse de las TIC en actividades de clase, entre otros.

Esto tiene como finalidad el conocer si estos docentes son conscientes de las ventajas de estas nuevas tecnologías en las clases de lengua y la aplicación de estos recursos tecnológicos en sus cursos y en qué forma. Por consiguiente, se recomendarán aspectos relevantes que 
URL: http://www.una.ac.cr/educare

deben ser contemplados cuando se incorporan las TIC a la enseñanza de una segunda lengua, y se aportarán algunas ideas sobre estrategias metodológicas con el uso de TIC, y su inclusión en el planeamiento y aplicabilidad en la clase.

De veinticinco copias del cuestionario repartidas entre los docentes de esta Escuela, se recibieron diecinueve completas; la información se sistematizó y analizó de la siguiente manera:

Al cuestionárseles si utilizaban algún recurso tecnológico cuando planean sus clases de lengua, dieciséis contestaron que sí los emplean en sus procesos de enseñanza. Esto evidencia que la mayoría de los docentes están conscientes de lo que representa un "buen saber hacer pedagógico" para mejorar la calidad de la formación que imparten.

Sin embargo, cuando se indagó sobre la forma en que aprovechan algún recurso tecnológico cuando planean sus clases de lengua, trece de los profesores tuvieron respuestas como buscar material adicional, ver películas o explicar temas. El resto dieron respuestas como uso de los estudiantes, desarrollar contenidos, comprensión gramatical y auditiva, y editar trabajos de los estudiantes. Este hecho evidencia el poco aprovechamiento de todas las posibilidades que las TIC pueden ofrecer en las clases de lengua y los beneficios que estas representan para sus estudiantes, dado que la implementación de las TIC permite a los docentes la diversificación de métodos y la promoción de un aprendizaje innovador y atractivo. Las respuestas obtenidas al respecto, se observan en la Figura 1.

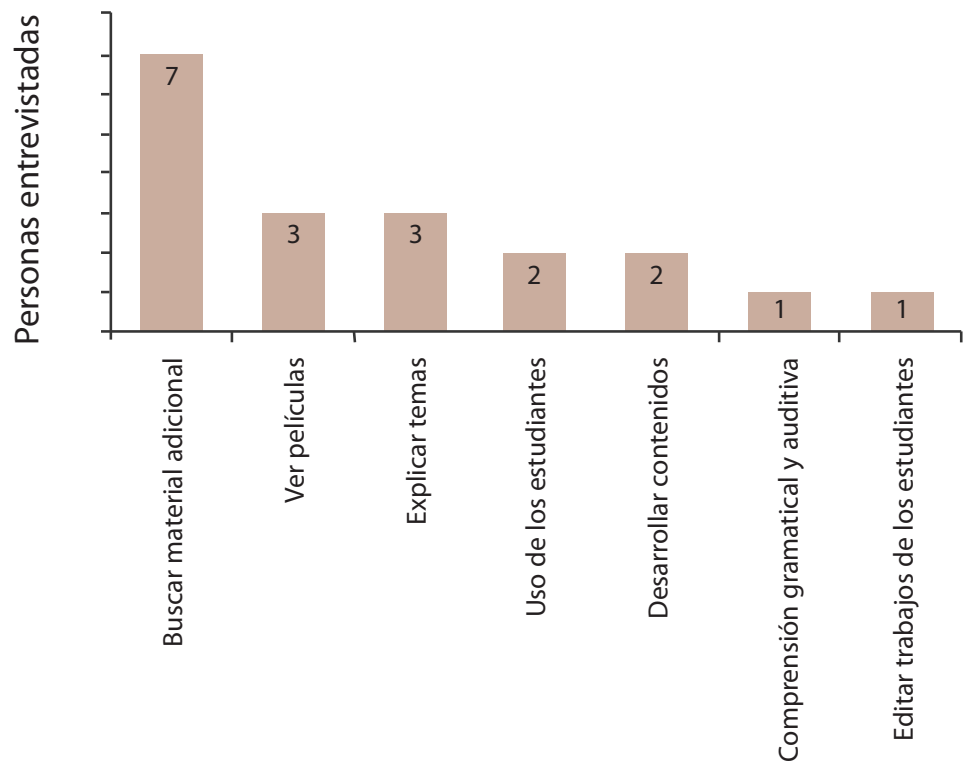

Formas

Figura 1. Formas de uso de los recursos tecnológicos al planear las clases de lengua 
URL: http://www.una.ac.cr/educare

CORREO: educare@una.cr

Seguidamente, se les pidió nombrar los cursos en los cuales usan las TIC y las respuestas se presentan en la Figura 2. Ocho de ellos las aplican en las clases orales, cinco personas las usan en todos los cursos que imparten y el resto en cursos de escritura, lectura, literatura o pronunciación de las consonantes, maestría, y comunicación. Esto demuestra que estas herramientas son empleadas por estos profesores solamente para estimular las competencias de escucha de los estudiantes a través del uso de películas, videos, discos compactos de música y ejercicios de escucha en las actividades planeadas en sus cursos, aspecto importante, pues esto introduce el uso de la herramientas tecnológicas en el planeamiento del currículo. Algunos docentes ignoran la existencia de otras formas de estimular las diferentes competencias de la lengua tales como blogs, conversaciones en Skype con nativos, el uso de chats, google docs, o redes sociales, que pueden ayudar de una forma innovadora y efectiva al estudiantado a mejorar sus habilidades lingüísticas.

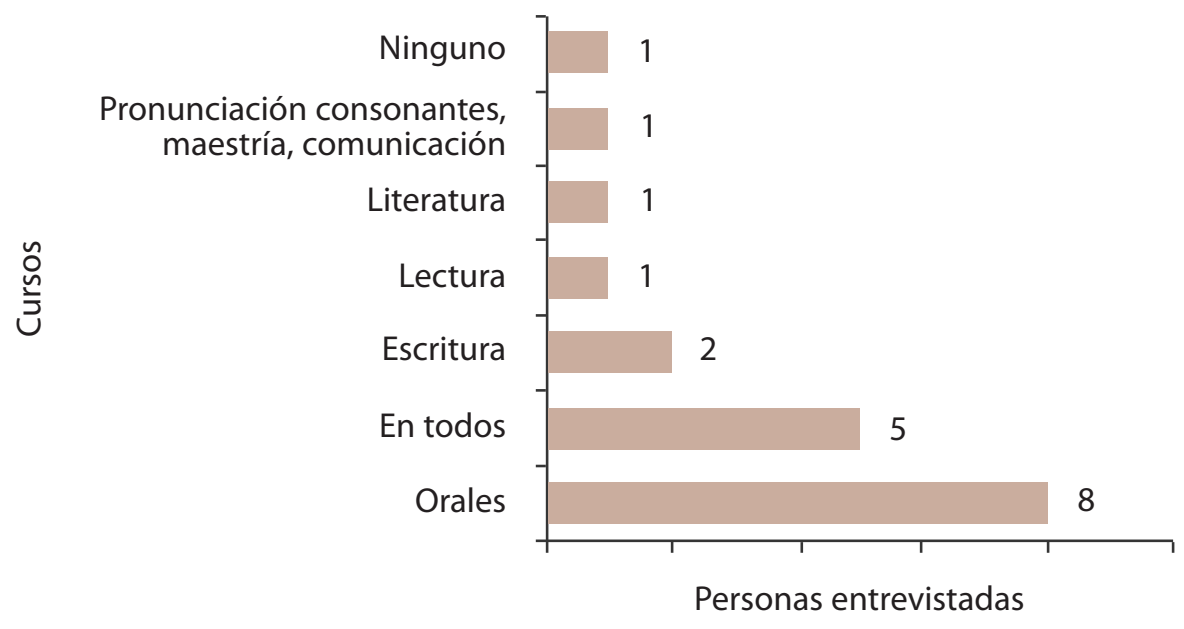

Figura 2. Cursos en que se utilizan las TIC

Cuando se les pidió su opinión sobre si al manejar las TIC en las clases, estas herramientas modifican sus estrategias de enseñanza, curiosamente la mayoría, dieciséis en total, no respondió.

No obstante, manifiestan que las TIC facilitan los procesos de enseñanza y aprendizaje de una lengua; la gran mayoría, diecisiete entrevistados, respondió en esos términos a la encuesta y agregó comentarios tales como: "ofrecen material complementario, hacen las clases más dinámicas, la información se sintetiza en una sola fuente, ofrecen interacción y conocimiento, etc.", según se muestra en la Figura 3. Este es un aspecto positivo que refleja que los profesores conocen las ventajas de las TIC en las clases de lengua y sus beneficios, a pesar de que nos las emplean en su totalidad. 
URL: http://www.una.ac.cr/educare CORREO: educare@una.cr

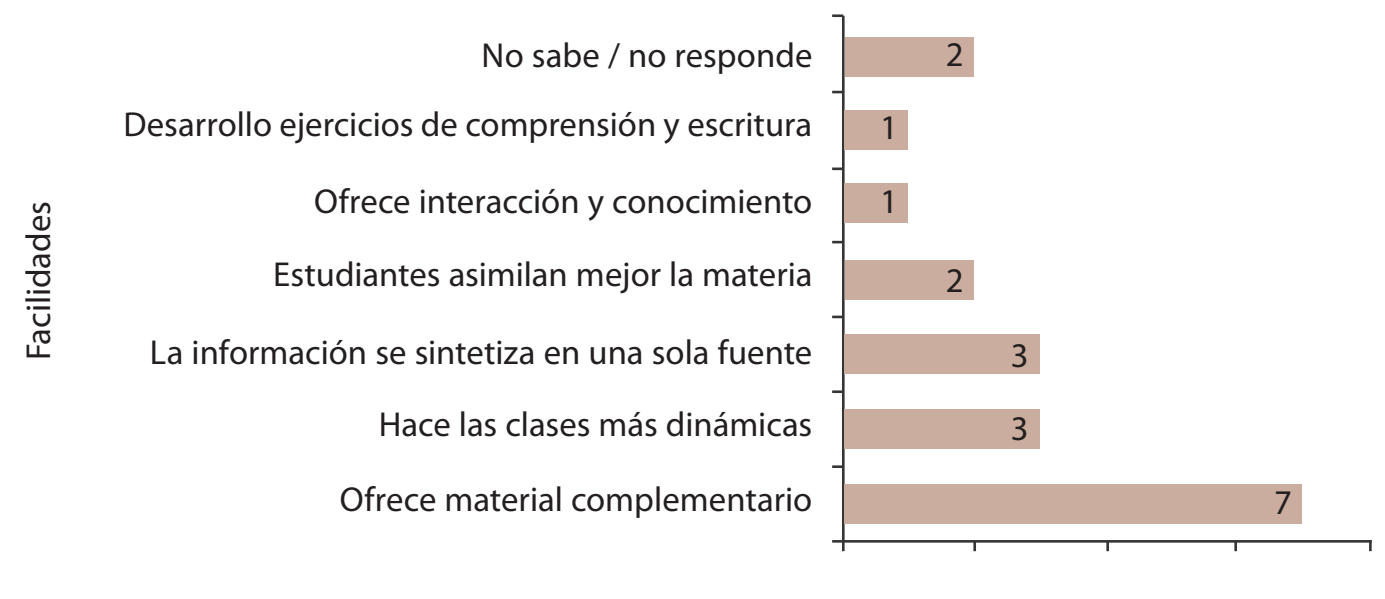

Personas entrevistadas

Figura 3. Facilidades que ofrecen las TIC en el proceso de enseñanza y aprendizaje de una segunda lengua

Igualmente, quince docentes manifiestan, en sus respuestas, ser conscientes de que el profesorado de lengua debe adaptar sus métodos de enseñanza al uso de las TIC, para ayudar al grupo estudiantil a ser innovador y analítico.

Sin embargo, al describir las actividades que realizan en la clase de lengua utilizando las $\mathrm{TIC}$, catorce entrevistados, el $74 \%$, nombran algunas ya tradicionales, como presentaciones orales, creación de videos y ver películas, investigación y explicaciones en clase; y muy pocos, solo tres docentes, que representan el $16 \%$, mencionan el uso de foros, chats u otros. Una apreciación que no refleja sus cambios de actitud a pesar de las opiniones favorables que ellos expresan. Lo anterior se presenta en la Figura 4.

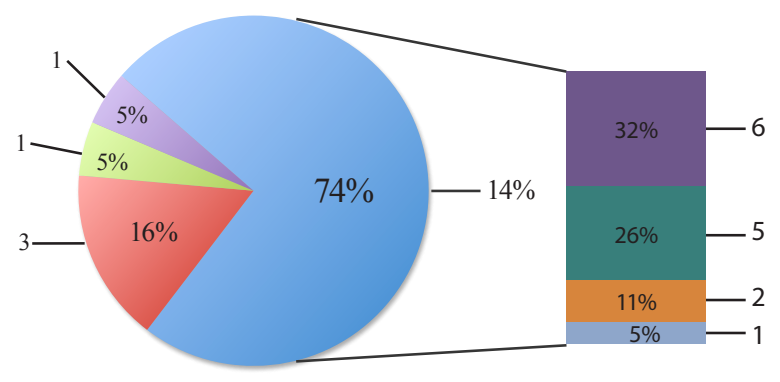

Figura 4. Actividades que se realizan en la clase de lengua utilizando las TIC
Escritura (foros, chats, etc.)

Ninguna

No responde

Presentaciones orales

Creación de videos y ver películas

Investigación

Explicaciones en clase 
URL: http://www.una.ac.cr/educare

CORREO: educare@una.cr

Sobre las ventajas de servirse de las TIC en la clase, según se observa en la Figura 5, trece mencionan como ventajas principales el que con estos recursos tecnológicos tienen acceso a material actualizado para planear actividades que ayuden a los estudiantes a desarrollar su creatividad o que brindan mayor motivación y creatividad. Por consiguiente, estas herramientas son, en su mayoría, visualizadas como un buscador de material nuevo para complementar sus clases de lengua, sin tomar en cuenta otras características positivas de las TIC en la enseñanza.

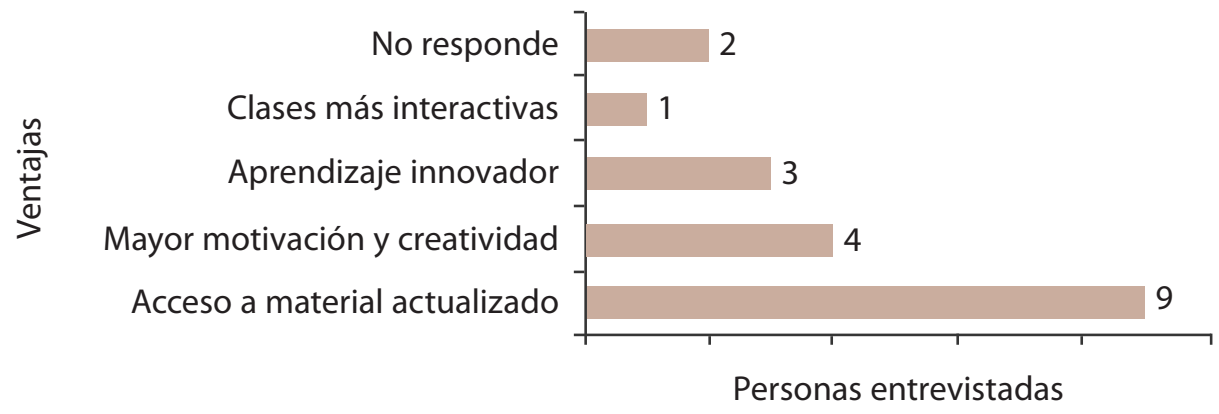

Figura 5. Ventajas de utilizar las TIC en las clases de lengua

Por su parte, doce docentes, el $63 \%$, opina que las competencias lingüísticas del estudiantado han mejorado al utilizar TIC en las actividades de clase. Por el contrario, un $26 \%$ expresa que no han mejorado, según se observa en la Figura 6.

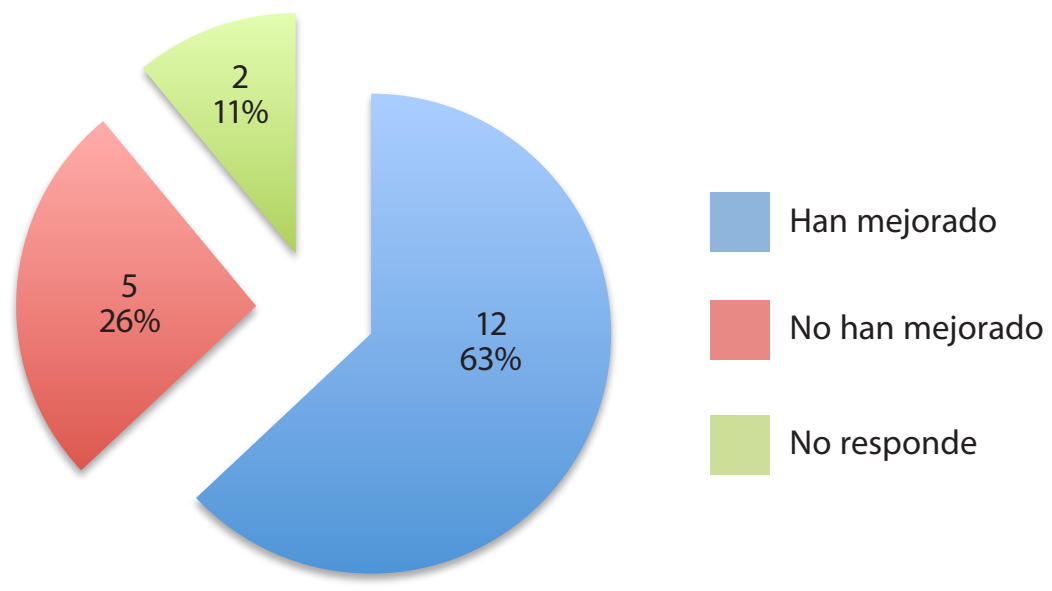

Figura 6. Mejora de las competencias lingüísticas de los estudiantes al usar TIC 
Al preguntárseles cuáles son las competencias que mejoraron con el uso de las TIC, según se muestra en la Figura 7, cuatro indicaron que el interés en las actividades de clase, cuatro que la competencia oral, dos señalaron que el trabajo cooperativo y una que la competencia auditiva. Uno mencionó las cuatro competencias. Por su parte, cinco respondieron que ninguna ha mejorado. Estos resultados indican que un grupo de docentes aún no está convencido de que los estudiantes progresen.

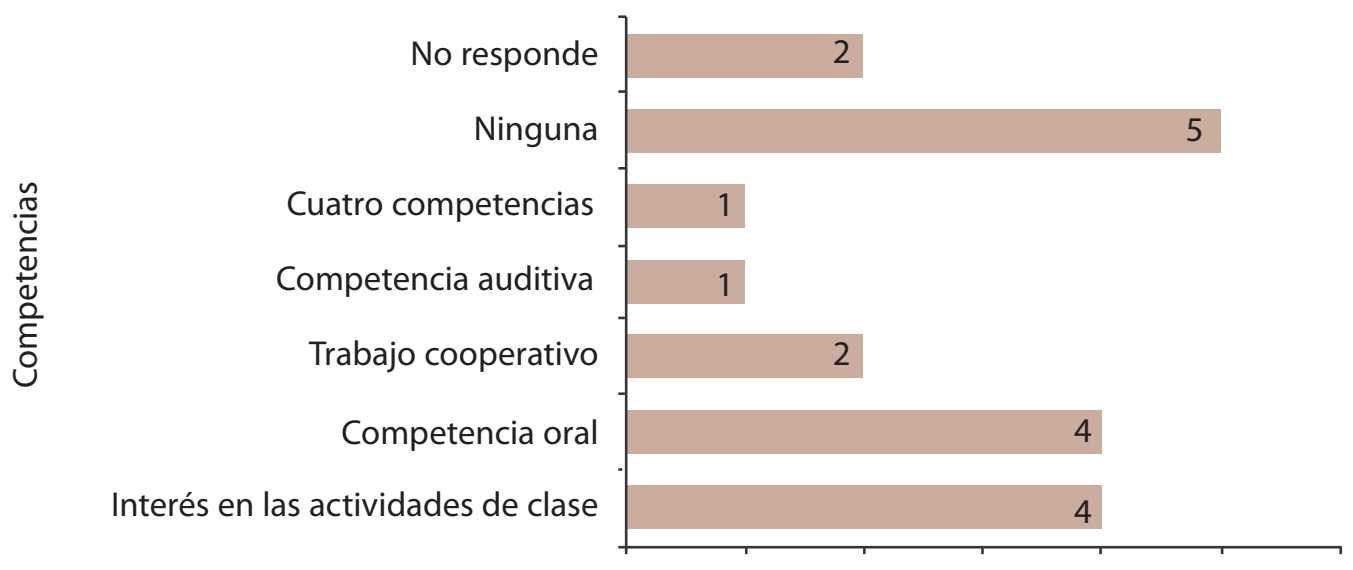

Personas entrevistadas

Figura 7. Competencias lingüísticas de los estudiantes que mejoran al utilizar las TIC

\section{Discusión}

Tradicionalmente se ha visualizado el salón de clase como un lugar donde dos participantes -docente y el estudiantado- interactúan durante cada sesión. En esta perspectiva, el rol docente es protagónico y portador del conocimiento absoluto, el cual debe trasmitir a sus aprendientes. Por otro lado, los estudiantes y las estudiantes tienen el rol de oyentes y participantes pasivos. Estos roles se han consolidado a lo largo de todo el proceso de aprendizaje, independientemente del tema en estudio, la carrera o la especialidad.

El proceso de aprendizaje de una segunda lengua no ha sido la excepción a este consolidado rol. Si analizamos objetivamente los métodos de enseñanza usados por muchos profesores de lengua, se puede determinar cómo el método tradicional de escuchar, repetir y completar tiene un rol protagónico en cada una de las sesiones en el salón de clase. Por tanto, con el objetivo de incorporar elementos innovadores y actuales, se requiere un cambio en la metodología y planeamiento de los temas de estudio.

Este cambio puede ser realizado si se incorporan las TIC como herramientas efectivas y prácticas para desarrollar actividades tanto escritas como orales en el aprendizaje de una segunda lengua. 
URL: http://www.una.ac.cr/educare

CORREO: educare@una.cr

A continuación, se mencionarán cuatro aspectos relevantes que deben considerarse cuando se incorporan las TIC en la enseñanza de una segunda lengua.

\section{Enseñanza reflexiva}

El determinar cuáles aspectos deben ser analizados y mejorados no es una tarea fácil para el personal docente. Este debe poseer la suficiente madurez para observarse y descubrir cuáles estrategias metodológicas no son funcionales para cumplir los objetivos propuestos en el curso. De acuerdo con Richards y Lockhart (1998), ". . . los contextos en que los profesores trabajan tienen una importante influencia en la enseñanza, ya que, según la situación docente, los profesores han de adoptar papeles distintos..." (p. 93).

Normalmente, quien enseña lengua tiende a planear las diferentes actividades académicas que se desarrollarán durante el curso, de acuerdo con el tema en estudio. Esto conlleva que el planeamiento sea muy subjetivo y no apegado a las necesidades reales del estudiantado y su proceso de aprendizaje. Un planteamiento objetivo y eficaz requerirá que el docente incorpore estrategias metodológicas aplicadas en otros cursos con una temática parecida. Así, el aprender de los errores cometidos en la realización de ciertas actividades en el salón de clase ayudará a que muchos de los objetivos propuestos se cumplan satisfactoriamente.

Uno de los más importantes objetivos del profesorado de lengua debe ser lograr que sus estudiantes aprendan la lengua meta por medio de estrategias de enseñanza que los ayuden a comprender, analizar y utilizarla en una forma clara y efectiva. Esto, con el objetivo de contextualizarla y actualizarla de acuerdo con el dialecto empleado. Por tanto, el planeamiento de cada una de las actividades debe ser focalizado en la incorporación de métodos efectivos tales como actividades interactivas grupales e individuales. Dichas actividades deben incorporar métodos tradicionales y métodos innovadores, tales como los recursos tecnológicos (TIC).

\section{Los equipos informáticos}

La utilización de software o programas especializados para la enseñanza de una lengua requiere que el personal docente tenga un conocimiento previo de estos y de su manejo en el salón de clase. En muchas ocasiones, el profesorado no posee ni el conocimiento ni la preparación para aprovecharlos efectivamente en cada uno de los cursos que se imparten en la enseñanza de una segunda lengua. En su artículo Apuntes metodológicos acerca de la enseñanza de lenguas asistida por ordenador, Pérez (2002) enumera una serie de programas tales como procesadores de textos (ejemplo: Microsoft Word, Open Office), presentaciones (Impress, Power Point), diccionarios electrónicos: online o offline, CD-Rom multimedia, programas de autor (por ejemplo: Hotpotatoes, Clic), plantillas en la Web, navegadores (como: Explorer, Firefox, Mozilla), pizarra electrónica (ej.: smartboard, ebeam). Estos programas ayudan a desarrollar destrezas lingüísticas orales y escritas en una forma clara y fluida. 
Sin embargo, estos programas no deben ser una herramienta que sustituya una clara y precisa explicación sobre el tema en estudio, pero pueden ser de gran ayuda para explicar el tema en una forma más creativa y práctica. Además, estos cuentan con actividades orales y escritas que permiten al estudiantado monitorear su desempeño y evolución lingüística en la lengua meta.

\section{Diversidad estudiantil}

Adquirir la lengua materna es un proceso natural e inherente que solo requerirá que el hablante esté en contacto con esta desde su infancia. Dicho proceso no es forzado o impuesto por factores externos tanto sociales como económicos. Sin embargo, el aprendizaje de una segunda lengua obliga al aprendiente a seguir un proceso no natural e impuesto. Por esto, el profesor de lengua no debe visualizar a los aprendientes como individuos que aprenden en una forma única y absoluta. Ellos evidenciarán diferencias significativas en su proceso de aprendizaje y el docente debe reconocerlas y adaptar su metodología a estas.

Cañarte y Pibaque (s. f., p. 1), citando a Deborah Healey, plantean:

Según Deborah Healey, académica de la Universidad de Oregón, el uso de las TIC en el desarrollo de la expresión oral en idioma inglés, [sic] busca cultivar el sentido de responsabilidad social en sectores vinculados con el desarrollo científico tecnológico y la innovación del siglo XXI. A través del lenguaje es posible la formación de individuos capaces de expresar claramente sus ideas, comprender el complejo mundo contemporáneo, profundizar en las ciencias, la técnica y el arte y realizar su actividad laboral de forma crítica y creadora".

No obstante, este desarrollo puede ser entorpecido por diversos aspectos negativos en su proceso de aprendizaje. Healey revela que la calidad de la comunicación se ve disminuida por manifestaciones como: inseguridad para hablar otro idioma, poco interés en desarrollo de destrezas, la no evidencia de práctica en la comunicación, la falta de motivación, y la tendencia a traducir al español.

Existen varias formas de adaptar los objetivos a la diversidad estudiantil y herramientas que ayuden al profesorado de lengua a lograr su mayor objetivo: el aprendizaje de la lengua meta. Entre estas se encuentra la incorporación, en el plan de curso, de diferentes actividades en las que se requiera incorporar los recursos informáticos manejados diariamente por los estudiantes: redes sociales, Skype, foros, blogs, etc. 
URL: http://www.una.ac.cr/educare

CORREO: educare@una.cr

\section{Estrategias metodológicas}

Durante el proceso de aprendizaje de una segunda lengua, el cuerpo docente se vale de actividades que solamente involucran la escritura y las repeticiones orales como parte de su estrategia metodológica. Sin embargo, estas son, en muchas ocasiones, percibidas por los aprendientes como aburridas o poco motivadoras. Así, la implementación de actividades orales, escritas y auditivas debe ser innovadora y motivar a los estudiantes a explorar y desarrollar sus capacidades lingüísticas para poder aprender la lengua meta.

Algunas de las actividades sugeridas a continuación explotan las TIC como eje central en el cumplimiento de objetivos y el desarrollo de habilidades lingüísticas:

a. Skype. Esta es una de las herramientas tecnológicas más utilizadas por los hablantes nativos y no nativos de una lengua. Por lo tanto, funciona como un método para establecer vínculos entre individuos de diferentes países cuyo único objetivo es poder aprender y practicar la lengua meta de modo oral. Además, esta es una forma de aprender no solamente la lengua en una manera informal, sino también permite comprender y recurrir a frases idiosincráticas de una lengua.

b. Foros. Las actividades escritas requieren que los estudiantes se identifiquen con el tema que se desarrollará. Por esto, el uso de foros como una herramienta para poder discutir en una forma escrita sobre temas relacionados con el curso resulta interesante para los educandos. Esto ayuda al empleo de la gramática en una forma contextualizada y permite el intercambio de ideas entre los diferentes participantes.

c. Blogs. A través de los blogs, los estudiantes son capaces de interactuar con hablantes nativos o aprendientes de una segunda lengua. Como resultado, ellos podrán usar un estilo escrito más informal de acuerdo con el grupo, jerga o variación dialectal.

d. Movie/Video creators. Esta herramienta tecnológica permite que los estudiantes tanto en forma grupal como individual realicen películas o videos. Esto potencializa el desarrollo de habilidades escritas y orales, al escribir diálogos o realizar grabaciones. Además, los aprendientes podrán escuchar diálogos pronunciados en diferentes dialectos, lo cual ampliará su variabilidad dialectal y su cultura en general sobre hablantes de la lengua inglesa.

e. Youtube. Esta herramienta es una de las formas más populares de aprender y divulgar una lengua y sus diferentes variaciones dialectales. Las ventajas de youtube son el poder lograr que un mensaje en la lengua meta sea trasmitido en un tiempo corto y sea recibido por una gran cantidad de usuarios de la red. Por medio de youtube, los estudiantes pueden desarrollar habilidades en el uso del lenguaje corporal, técnicas de conversación, compartir el resultado de una investigación y compartir problemas que aprendientes de una segunda lengua enfrentan en su proceso de aprendizaje. Finalmente, esta puede servir como una forma de crear un entorno más actualizado del uso de la lengua meta y su proceso de aprendizaje. 


\section{Ventajas y desventajas del uso de las TIC}

Existen muchas ventajas en la aplicación de las TIC para la enseñanza de una segunda lengua. El software libre y equipos informáticos son promovidos por organismos internacionales, tales como la UNESCO, en todos los países del mundo. El objetivo es acortar la brecha entre países pobres y aquellos con gran riqueza y un mayor desarrollo. Morán (2012) agrega ventajas tales como la forma en que los niveles de conocimiento son adquiridos y los modelos pedagógicos innovadores que ellas estimulan.

Sin embargo, esta autora menciona algunas desventajas: la tensión y el desconocimiento de los facilitadores del aprendizaje en cuanto al uso de las TIC, y la producción de contenidos.

Por lo anterior, es relevante mencionar que las TIC son un instrumento que ayuda al personal docente a planear y desarrollar sus lecciones. Este no es el único elemento disponible para la enseñanza de una segunda lengua; el recurso tecnológico debe ser adaptado de acuerdo con el contexto social, humano y metodológico.

\section{Conclusiones}

El uso de las TIC en la enseñanza superior ha sido un tema relevante en los últimos años. Esto ha generado un boom a nivel de docentes y especialistas en la enseñanza de un área en particular. La implementación de las TIC en la enseñanza de una segunda lengua no ha sido la excepción. Actualmente, el profesorado de segunda lengua es invitado a aprender e implementar instrumentos tecnológicos en el currículo de sus cursos. Al respecto, el cuestionario proporcionó información relevante, la cual se brinda en las conclusiones que a continuación se mencionan:

a. El personal docente reconoce que un "buen saber hacer pedagógico" ayuda a mejorar la calidad de los cursos impartidos. No obstante, los recursos tecnológicos -tales como, videos, películas y textos- son solamente utilizados para la búsqueda de información y como material de apoyo. Esto no permite que las diversas herramientas tecnológicas brinden diversidad en las actividades planeadas y estimulen el desarrollo mental y creativo de los educandos en una clase de lengua.

b. Además, se evidenció que el profesorado emplea las TIC principalmente en cursos orales para estimular la habilidad auditiva y comprensión de dialectos. Esta limitación en su uso no permite que el profesor de lengua incorpore las TIC como herramientas tecnológicas que también pueden estimular la escritura; la socialización con hablantes nativos; el intercambio de ideas y la discusión de estas por medio de blogs, foros o redes sociales. Estos usos potencializarían las habilidades lingüísticas de sus aprendientes. 
URL: http://www.una.ac.cr/educare

CORREO: educare@una.cr

c. Claramente, se muestra el reconocimiento, por parte de los profesores y profesoras de lengua, de que las TIC ofrecen material complementario actualizado, el cual permite que las clases sean menos tradicionales y más innovadoras. No obstante, este reconocimiento no es suficiente para que estas herramientas tecnológicas sean incorporadas completamente en el currículo de la clase.

d. Finalmente, quienes han incorporado las TIC en sus actividades en la clase consideran que las competencias lingüísticas de sus estudiantes han mejorado a través del trabajo cooperativo y el uso de ejercicios o actividades auditivas. Cabe mencionar que la implementación de TIC en el planeamiento de las actividades a realizarse en la clase no es una práctica común entre el personal docente de lengua. Este colectivo profesional todavía recurre a las herramientas tecnológicas rudimentariamente o las aprovecha en muy pocas ocasiones.

Por esto, ciertos criterios deben ser mencionados para comprender que dicha implementación es una necesidad para el buen desempeño docente:

a. Los centros de educación superior en que se imparten carreras de enseñanza de lengua requieren que la formación docente se actualice y maneje recursos tecnológicos innovadores y versátiles. Por consiguiente, este requerimiento continuo hacia la actualización y conversión tecnológica de los profesores y profesoras deberá estar acompañado de estrategias que les permitan desarrollar sus habilidades para enfrentar la gran tarea de planear clases que incorporen los TIC en el aula. Además, se les debe capacitar para poder enfrentar las barreras tecnológicas y lo cambiante de esta área.

b. El diseño de la enseñanza es una herramienta primordial para lograr el éxito en el aprendizaje de una segunda lengua. La implementación de programas tecnológicos traerá cambios innovadores en los programas que se apliquen en las diferentes carreras de la enseñanza de lengua. Así, el uso de laboratorios de lengua, de paquetes interactivos, de ayuda a distancia por medio de programas especializados u otro material de apoyo despertarán el deseo por aprender una segunda lengua y brindarán estrategias de enseñanza efectivas y atractivas para ambos, docentes y estudiantes.

c. Por lo tanto, el aula pasará de ser un lugar donde el conocimiento se brinda en una forma vertical, a un entorno en el cual tanto profesorado como alumnado aprenderán a interactuar y desarrollar destrezas. El personal docente podrá innovar sus estrategias de enseñanza a través del planeamiento de clases donde el desarrollo auditivo, oral y escrito sean potenciados en una forma progresiva. 
Además, el aprendiente tendrá la oportunidad de desarrollar sus habilidades libremente y de acuerdo con sus capacidades y forma de aprendizaje. El uso de la Internet, paquetes interactivos, aulas virtuales, chats interactivos u otro recurso logrará que el aprendizaje de un segundo, tercer o cuarto idioma sea un proceso creativo en el cual el alumnado sea un participante activo y creador de su propio proceso de aprendizaje.

d. En los resultados de la encuesta se evidencia cómo el docente de segunda lengua tiene escaso conocimiento de las TIC y, por lo tanto, no las implementa en sus clases. Sin embargo, esto no debe ser un obstáculo para iniciar un proceso de aprendizaje y renovación de ideas y procesos. El docente no debe ser analfabeto funcional en un proceso globalizado; las TIC son y serán herramientas necesarias para la enseñanza de cualquier lengua y para el desarrollo de habilidades lingüísticas en la persona aprendiente. Por esto, el cuerpo docente debe ser un facilitador de conocimientos y estrategias e ir a la vanguardia de la tecnología.

Definitivamente, se está dando un impacto tecnológico muy fuerte en el uso de las diferentes herramientas (TIC) en la enseñanza de una segunda lengua, y cada vez más el personal docente es requerido a ser parte de este proceso innovador e inherente a la enseñanza que imparte. Así, cualquier docente que desee estar en concordancia con las nuevas tendencias debe recibir capacitación e incorporar lo aprendido en su currículo y estrategias metodológicas.

Finalmente, la incorporación de las TIC a su currículo y desempeño docente será fundamental en el desarrollo de las habilidades lingüísticas de sus estudiantes.

\section{Referencias}

Cañarte, J. W. y Pibaque, M. (s. f.). El uso de las tic en el desarrollo de la expresión oral en el idioma inglés de la educación superior. Recuperado de http://www.monografias.com/trabajos83/ doctora-ciencias-educacion/doctora-ciencias-educacion.shtml\#top

Contreras, N. (2008). La enseñanza-aprendizaje de lenguas extranjeras y las TICs: El caso del español como Lengua Extranjera (ELE). Iniciación a la Investigación, Especial, i-vii Recuperado de http://revistaselectronicas.ujaen.es/index.php/ininv/article/view/233/214

Fernández, R. R., Server, P. M. y Carballo, C. E. (enero, 2006). Aprendizaje con nuevas tecnologías paradigma emergente. ¿Nuevas modalidades de aprendizaje? EDUTEC. Revista Electrónica de Tecnología Educativa, 20, 1-24. Recuperado de http://edutec.rediris. es/Revelec2/revelec20/raul20.pdf 
URL: http://www.una.ac.cr/educare

CORREO: educare@una.cr

Manga, A-M. (diciembre, 2008). Lengua Segunda (L2) Lengua Extranjera (LE): Factores e Incidencias de Enseñanza/Aprendizaje. Revista Electrónica de Estudios Filológicos, 16(16) Recuperado de http://www.um.es/tonosdigital/znum16/secciones/estudios--10Ensenanza.htm

Morán, M. (18 de abril de 2012 ). Ventajas y desventajas del uso de las tic. [Mensaje en un blog]. Recuperado de http://excelenciaeneducacion.blogspot.com/2012/04/ventajas-ydesventajas-del-uso-de-las.html

Pérez, M. I. (2002). Apuntes metodológicos acerca de la enseñanza de lenguas asistida por ordenador (ELAO). GRETA Revista para profesores de inglés, 10(1), 55-61. Recuperado de http://www.freewebs.com/cfc03/apuntesmetodologicos.pdf

Richards, J. C. y Lockhart, Ch. (1998). Estrategias de reflexión sobre la enseñanza de idiomas. Cambridge University Press.

Salinas, J. (noviembre, 2004). Innovación docente y uso de las TIC en la enseñanza universitaria. Revista Universidad y Sociedad del Conocimiento, 1(1), 1-16. Recuperado de http://www. uoc.edu/rusc/dt/esp/salinas1104.pdf

UNESCO. (2004). Las Tecnologías de la información y comunicación en la formación docente: Guía de planificación (Trad. F. Trías y E. Ardans). Montevideo: Ediciones Trilce. Recuperado de http://unesdoc.unesco.org/images/0012/001295/129533s.pdf

Vélez, D. E, Vidal, M. y Yamá, D. (2010). Importancia de las TICS en la enseñanza de los idiomas: Beneficios [Diapositivas en PowerPoint]. Recuperado de http://es.scribd.com/ doc/28772610/IMPORTANCIA-DE-LAS-TICS-EN-LA-ENSENANZA-DE

\section{Cómo citar este artículo en APA:}

Pizarro, G. y Cordero, D. (2013). Las TIC: Una herramienta tecnológica para el desarrollo de las competencias lingüísticas en estudiantes universitarios de una segunda lengua. Revista Electrónica Educare, 17(3), 277-292. Recuperado de http://www.revistas.una.ac.cr/index.php/EDUCARE/issue/current

Nota: Para citar este artículo en otros sistemas puede consultar el hipervínculo "Como citar el artículo" en la barra derecha de nuestro sitio web:

http://www.revistas.una.ac.cr/index.php/EDUCARE/index 